\title{
Benedetto Varchi e l'averroismo
}

\author{
di Luca Bianchi*
}

Abstract: Benedetto Varchi and Averroism. The article examines the role played by Averroes and the Averroist tradition in Benedetto Varchi's philosophical works. The first part clarifies why Varchi defines Dante as an "Averroist" and reconstructs his original reading of the first verse in Paradise (Paradiso). The second part argues that Varchi's plan to vernacularize philosophy, often interpreted as an attempt to "democratize" knowledge, should not prevent us from recognizing that he emphasizes the moral and intellectual differences existing among human beings. Though following Aristotle in saying that "all men by nature desire to know", Varchi shows no qualms in acknowledging that most of them cannot fulfil this desire and, referring to Averroes and Galen, claims that because of their complexio most people cannot understand philosophy.

Keywords: Benedetto Varchi, Dante Alighieri, Averroism

\section{Benedetto Varchi, il Dante "averroista" e l'incipit del Paradiso}

La tesi dell'averroismo di Dante è universalmente associata al nome di Bruno Nardi. Fu lui, infatti, che nel combattere la "leggenda" che faceva del sommo poeta un fervente tomista sostenne che il Convivio e la Monarchiadue testi che riteneva vicinissimi non solo contenutisticamente ma anche cronologicamente - sarebbero profondamente influenzati da idee risalenti a Averroè, e che "residui" di quest'influenza sarebbero rintracciabili persino nel $\mathrm{Pa}$ radiso ${ }^{1}$. Di recente molti studiosi hanno rifiutato lo schema evolutivo sotteso a

*luca.bianchi@unimi.it: professore ordinario di Storia della filosofia medievale presso l'Università degli studi di Milano. Articolo proposto nel maggio 2020, accettato per la pubblicazione nel luglio 2020. Questo contributo è stato portato a termine nell'ambito delle ricerche dell'unità di Milano del progetto PRIN 2017 «Averroism. History, Developments and Implications of a Crosscultural Tradition» (PI: Pasquale Porro; 2017H8MWHR).

1. Sull'attività di Bruno Nardi e il suo modo di concepire la funzione dell'averroismo nel pensiero medievale, in particolare in Dante, si vedano Gregory 1968, Stabile 1991, Falzone 2013, Fioravanti 2019.

Rivista di storia della filosofia, n. 4 2020, Issn 0393-2516, Issn-e 1972-5558

DOI: 10.3280/SF2020-004001 
quest'interpretazione del pensiero dantesco e si sono chiesti se la categoria storiografica "averroismo", introdotta nel 1852 da Ernest Renan nel celebre studio Averroès et l'averrö̈sme, sia ancora valida e possa essere utilmente impiegata per comprendere il percorso intellettuale di Dante. Avendo contribuito a questo dibattito ${ }^{2}$, vorrei qui proporre qualche osservazione intorno a un semplice interrogativo: fu davvero Nardi il primo a ipotizzare un rapporto fra Dante e l'averroismo?

La risposta è sicuramente negativa. Nel 2017 Catherine König-Pralong ha richiamato l'attenzione sul fatto che nella sua Dantes Trilogie, del 1869, Karl Witte aveva sostenuto che, prima di divenire il poeta cristiano della Commedia, Dante sarebbe stato "fuorviato" dall'aristotelismo arabo diffusosi nel corso del secolo XIII, di modo che nel Convivio e nella Monarchia - da lui datata addirittura prima del 1300 - avrebbe finito per accogliere una metafisica ispirata a Averrò̀ . $^{3}$

Una valutazione ben diversa di queste due opere aveva dato tre decenni prima Pompeo Azzolino, nella sua Introduzione alla storia della filosofia ai tempi di Dante. Si tratta di un lavoro del tutto dimenticato, che meriterebbe un esame approfondito e che, anche a una rapida scorsa, risulta assai interessante per una molteplicità di motivi ${ }^{4}$. Pur muovendo dall'anacronistico assunto che Dante volesse elaborare un «sistema filosofico» compiuto, Azzolino aveva fra l'altro intuito quel che molti dopo di lui - sino a Nardi - non vollero riconoscere, cioè che la riflessione dantesca si alimentò a una pluralità di fonti, antiche, patristiche e medievali. Fra queste Azzolino non escludeva potessero esservi anche gli scritti di pensatori legati alla «scuola degli Averroisti»:

Oserei anche affermare che sebbene molti timidi pensatori del secolo decimoquarto strepitassero contro la scuola degli Averroisti, a Dante non dispiacesse l'arditezza di alcune opinioni di costoro, le quali al suo sistema, in che la religione e la fede poste al loco loro diventavano invulnerabili, non potevano nuocere; al suo fine d'altronde d'indebolire il despotismo Teocratico non facevano che appianare la via ${ }^{5}$.

Azzolino purtroppo non chiariva che cosa intendesse per «scuola degli Averroisti». A distanza di quasi due secoli abbiamo idee assai più precise sulla ricezione dell'Averroes latinus e sulla profonda influenza da esso esercitata sui contemporanei di Dante, ma sappiamo anche che l'esistenza di una vera e propria "scuola averroista" è quantomeno controversa". Bisogna del resto ricordare che, diversamente da Witte, Azzolino scrisse il suo lavoro

2. Cfr. Bianchi 2013, Bianchi 2015, Bianchi 2018b, Bianchi 2019.

3. Cfr. König-Pralong 2017, pp. 22-25.

4. Cfr. Azzolino 1839. Un mio contributo sull'opera di Azzolino è in fase di stesura.

5. Azzolino 1839, p. 89. In questa e nelle successive citazioni i corsivi sono miei, salvo diversa indicazione. D'autore sono i corsivi dei versi boeziani e danteschi riprodotti all'interno dei passi citati.

6. Sulla nozione di "averroismo", la sua genesi e la sua successiva crisi la letteratura è ampia: mi limito a rimandare a Fioravanti 1966, Imbach 1991, Hasse 2007, Giglioni 2013, Bianchi 2015, pp. 71-78. 
prima della pubblicazione del saggio di Renan; e che in questo stesso saggio la nozione di "averroismo" era tanto centrale quanto irrimediabilmente vaga, al punto che Renan da un lato la applicava a pensatori molto diversi fra loro, operanti in vari paesi europei fra il XIII e il XVII secolo, dall'altro sosteneva che «le système désigné au moyen âge et à la Renaissance sous le nom d'averroïsme n'est que l'ensemble des doctrines communes aux péripatéticiens arabes» ${ }^{7}$.

Comunque sia, una prima conclusione è possibile. Riferimenti a una dipendenza di Dante da Averroè e dalla sua «scuola» si trovano già nella storiografia ottocentesca dove con Azzolino e Witte comincia ad emergere, pur da prospettive diverse, l'ipotesi cui Nardi avrebbe poi dato forma compiuta: quella secondo cui nel Convivio e nella Monarchia la distinzione fra verità filosofiche e verità di fede sarebbe l'espressione di un "razionalismo" di ispirazione "averroista" che, sul piano politico, si tradurrebbe nella separazione fra potere temporale e potere ecclesiastico. L'idea che Dante possa, in qualche modo, essere qualificato come "averroista", è però molto più antica e nasce in tutt'altro contesto. È infatti rintracciabile in Benedetto Varchi, una figura della quale proprio Nardi si interessò, mettendone in luce la dipendenza da Ludovico Boccadiferro $^{8}$, e che da una quindicina d'anni è oggetto di rinnovati studi, fra $\mathrm{i}$ quali spiccano quelli di Anna Siekiera, Salvatore Lo Re, Annalisa Andreoni, Simon Gilson, Marco Sgarbi e Dario Brancato9.

Nel 1543, introducendo le sue lezioni sul venticinquesimo canto del Purgatorio, Varchi assume un approccio interpretativo alternativo a quello neoplatonizzante di Cristoforo Landino, che pure è fra le sue fonti preferite ${ }^{10}$, e dichiara di voler spiegare Dante

seguitando sempre Aristotile, principe de' peripatetici, e il suo comentatore Averrois, $i$ quali due senza dubbio seguitò in questo luogo, e quasi tutti gli altri della Commedia

7. Renan 2002 (ristampa, con introduzione, della prima edizione del 1852), p. 77. Su Renan si soffermano tutti i lavori citati alla nota precedente: ma si veda in particolare Marenbon 2013.

8. Cfr. Nardi 1965, pp. 322-328. Il ruolo di Boccadiferro nella formazione filosofica di Varchi era segnalato già da Barbi 1890, p. 211; ma si veda la più ampia analisi di Pirotti 1971, pp. 78-81, 85-89 e Vasoli 2007, pp. 6-14. La profonda influenza esercitata da Boccadiferro sul commento varchiano alle Meteore è dimostrata da Gilson 2016; il debito di Varchi nei confronti di Boccadiferro nella trattazione del problema dei mostri è invece evidenziato da Montemagno Ciseri 2007, pp. 342-343. In Bianchi 2004, pp. 363-374 e Bianchi 2011 si mostra che Boccadiferro non può essere ridotto alla categoria "averroismo" e si segnala la compresenza nei suoi commentari aristotelici di elementi "scolastici" e umanistici.

9. Cfr. Siekiera 2007, Lo Re 2008, Andreoni 2012, Gilson 2012, Siekiera 2013, Andreoni 2014, Sgarbi 2014, Brancato 2018a, Brancato 2018b, Gilson 2018, pp. 115-125. Merita inoltre di essere consultato il lavoro, poco noto, di Devlieger 2005.

10. Sulla datazione di queste lezioni e la «venerazione» di Varchi per Landino si veda Andreoni 2012, pp. 93-101 (che, rifacendosi a Nardi, sottolinea anche la «fascinazione nei confronti di Boccadiferro»). Cfr. inoltre Gilson 2018, pp. 117, 120. 
e opera sua Dante medesimo, il quale fu grandissimo Peripatetico, se non quanto dalla Fede nostra e santissima religione cristiana gli fu vietato ${ }^{11}$.

Varchi presenta qui Dante come «grandissimo Peripatetico» e precisa che, fatte salve le verità di fede e alcune rare eccezioni, tanto nella Commedia quanto nell'insieme della «opera sua» egli seguì Aristotele e il suo commentatore per eccellenza. Il «grandissimo Peripatetico» è quindi un averroista, e la complementarità delle due qualifiche è esplicitamente enunciata nella decima e ultima lezione sul primo canto del Paradiso, tenuta nell'agosto del 1545. Secondo Varchi Dante si sarebbe infatti espresso «come grandissimo Peripatetico ed Averroista» quando, nell'incipit del Paradiso, definì Dio come «colui che tutto muove» ${ }^{12}$.

Per cogliere pienamente il significato di quest'affermazione a prima vista sorprendente bisogna anzitutto sottolineare che fra i commentatori, sia antichi sia moderni, Varchi si segnala per lo spazio eccezionalmente ampio che dedica a chiarire il significato di questo celeberrimo verso ${ }^{13}$. In effetti assai scarno era già l'autocommento dello stesso Dante nell'epistola a Cangrande che, in linea con convergenti passi del Convivio e della Monarchia, traduceva «di colui che tutto muove» con «Primi motoris» ma non offriva alcuna ulteriore spiegazione $^{14}$. Molti commentatori medievali e rinascimentali sono altrettanto sbrigativi e si accontentano di spiegare che Dio viene presentato come Primo Motore in quanto è causa prima del movimento di tutte le creature: l'unico aspetto rilevante è che spesso essi slittano con una disinvoltura un po' eccessiva

11. Varchi I, pp. 11-12, sui cui Andreoni 2012, p. 101 e Gilson 2018, pp. 116-117. Nell'interpretare il primo canto del Paradiso Varchi si meraviglia che Dante, «usato sempre di seguitare dove può la dottrina d'Aristotile e del suo grandissimo commentatore», accolga dai Pitagorici e da Platone l'idea della musica celeste: cfr. Varchi I, p. 329.

12. Varchi I, p. 400. La qualifica di Dante come «grandissimo Peripatetico ed Averroista» non era sfuggita a Nardi (cfr. Nardi 1965, p. 381), che però non si soffermò sui motivi che spinsero Varchi ad utilizzarla. Sulla caratterizzazione di Dante come «Peripatetico», diffusa fra i commentatori cinquecenteschi, si veda l'ampia analisi di Gilson 2012, che a p. 60 si riferisce proprio a Varchi; si veda anche Gilson 2018, p. 116. Per la datazione delle lezioni sul primo canto del Paradiso rimando a Andreoni 2012, pp. 155-156.

13. Un riferimento a Par. 1, 1 si incontra anche nella più tarda lezione sul diciassettesimo canto del Purgatorio, nel contesto di un'analisi della diffusione della bontà divina che è chiaramente ispirata al Liber de causis, pur non citato esplicitamente: cfr. Varchi I, pp. 161162. Senza riferirsi a questo specifico luogo, l'originalità dei commenti danteschi di Varchi è segnalata già da Barbi 1890, p. 212.

14. Azzetta 2016, pp. 378-379, con la nota al par. 53. Non ritorno qui sulla vexata quaestio dell'autenticità dell'epistola, a favore della quale proprio Azzetta ha portato argomenti molto convincenti, anche se non universalmente accolti come tali dai dantisti. L'identificazione fra Dio e il Primo Motore è costante in Dante: nel Convivio si dice espressamente: «chi mosse l'universo, cioè Dio» (III viii 22, ed. Fioravanti, p. 442). Nella Monarchia Dio viene qualificato come «primo motore» («in mente primi motoris, qui Deus est», II ii 2, ed. P. Chiesa, A. Tabarroni, p. 78); come «primo agente» («secundum intentionem primi agentis, qui Dues est», I viii 1, ed. P. Chiesa, A. Tabarroni, p. 32); come «unico motore» («ab unico motore, qui Deus est», I ix 2, ed. P. Chiesa, A. Tabarroni, p. 34). 
dalla funzione motrice a quella creatrice per sottolineare, con richiami al vangelo di Giovanni, che il Dio-Motore del Paradiso dantesco è il Dio cristiano, cioè l'onnipotente creatore che dà essere all'intera realtà ${ }^{15}$. Pietro Alighieri invece mette al centro della sua analisi proprio l'idea del Dio-Motore, insistendo sulla sua immobilità, ma non associa questa caratteristica alla Fisica e alla Metafisica di Aristotele, come sarebbe stato ovvio fare ${ }^{16}$, bensì a un'eterogenea molteplicità di fonti, fra le quali spicca il celebre e magnifico carme 9, O qui perpetua, del III libro della Consolazione della filosofia di Severino Boezio. Scrive Pietro:

et a remotis proemizando dicit, quod gloria, idest ore multorum celebrata laudatio, secundum Augustinum, illius qui totum movet, nec ipse movetur; testante Boetio dum dicit:

Stabilisque manens das cuncta moveri:

ad hoc Thomas etiam in primo: omne quod movetur, ab alio movetur, ut sol et alia; unde ne in infinitum eatur, necesse est quod ponatur aliquid movens immobile ${ }^{17}$.

Gli fanno eco nel Quattrocento Bartolomeo di Colle Val d'Elsa e nel Cinquecento, più estesamente, Cristoforo Landino, che coglie l'occasione per for-

15. Questa linea interpretativa attraversa l'intera tradizione esegetica e giunge, con una notevole ripetitività, sino ai nostri giorni. Per limitarsi a qualche esempio si vedano Iacomo della Lana (ENCD, vol. 3.3, p. 1695): «che tutto move. Questo primo motore si è Dio, lo quale move tutto, da chi è tutte le creature, sí com'è scritto in santo Ioanni, primo: “Omnia per ipsum facta sunt"»; l'Ottimo Commento (ENCD, vol. 6.3, p. 1328: «- che tutto move. Dio è primo motore, lo quale muove tutto; onde ne l'Evangelio di sancto Ioanni nel principio: "Tutte le cose sono facte per luil"»; Francesco da Buti (ed. C. Giannini, Pisa 1862, p. 11): «di Colui che tutto move, cioè di Dio lo quale ogni cosa muove et egli sta immobile, elli è primo motore [...] dunqua Idio primo motore e prima cagione di tutte le cagioni stando elli immobile, dunqua non vuole dire altro l'altore se non l'opera della chiara notizia di Dio la quale da ogniuno ène lodata: imperò che in tutta la creatura si cognosce distintamente, et in essa loda lo suo creatore: imperò che in essa riluce la notizia della onnipotenzia sua, della infinita sapienzia e della benivolenzia sua»; Niccolò Tommaseo (ed. U. Cosmo, Torino 1927, p. 7): «Muove: Joan. I, 3: "Tutte le cose per esso furono fatte". August.: "Chi tutto muove, nè egli è mosso"»; Isidoro del Lungo (ed. Firenze 1927, p. 651): «"colui": Dio, creatore e motore di tutto quanto è»; Manfredi Porena (cito da https://dante.dartmouth.edu/search_view.php?doc=194763010010\&cmd=gotoresult\&argl=5): «Colui che tutto move: Dio, motore primo dell’Universo: il moto è considerato come una sola cosa con la vita e l'esistenza».

16. Sappiamo del resto che la tesi che Dio è il Primo Motore che «tutto muove» era ben nota e veniva attribuita ai «filosofi» anche al di fuori del contesto delle università e degli studia degli Ordini religiosi. Per limitarsi a un esempio, nel predicare ai Fiorentini in anni che precedono la stesura del Paradiso, Giordano da Pisa affermava: «I filosofi danno del movimento tre belle regole [...]. E però Idio immobile tutte le cose muove, e questa è la terza regola, la quale danno i filosofi nel movimento, che dicono ch'è mistieri che ogne movimento sia da cosa immobile, che non si muti». Cfr. Quaresimale fiorentino (1305-1306), ed. C. Delcorno, pp. 132-133.

17. Cito dall'ed. V. Nannucci, Firenze 1845, pp. 543-544. È utile ricordare che il v. 3 dell'O qui perpetua era già stato usato nell'Ottimo Commento (ENCD, vol. 6.3, p. 1743) nello spiegare Par. 24, 130-132, ove Dio «tutto '1 ciel move, non moto». In proposito cfr. Lombardo 2013, pp. 501-502, che mostra la lunga fortuna di questa tradizione esegetica. 
nire una sua personale rilettura della "prima via" dell'Aquinate per dimostrare l'esistenza di Dio:

la gloria, la gloriosa opera, et è l'opera di Dio l'universo, di colui che tucto mиo$v e: i$ di Dio, et è color rhetorico decto circuitione, che è quando quello che si può dire in una parola si dice in più, tucto muove: onde Boetio: "Stabilisque movens [sic!] das cuncta moveri". Solo adunque lui è stabile; questo così pruova Tomaso aquinate, ogni cosa che è mossa conviene che sia mossa da altri, chome è sole luna et gl'altri. El perché acciò che non habbiamo a procedere in infinito è necessario porre uno motore inmobile. Iddio adunque inmobile muove, et tanto significa chi tucto muove quanto Iddio, imperò che lui essendo stabile et inmobile è primo motore; lui muove gl'angeli et gl'angeli e cieli et e cieli con loro revolutioni inducono giù nel mondo diversi effecti. Adunque Iddio è primo motore et prima cagione di tucte le cagioni ${ }^{18}$.

Nella sua prima lezione sul primo canto del Paradiso Varchi, offrendone un commento letterale, si riallaccia alla tradizione esegetica che identifica Primo Motore e Creatore e spiega che «non vuole significare altro il poeta se non che 'l Motore, ciò è Dio, dà l'essere e 'l vivere a tutte le cose di tutto il mondo». Tuttavia egli richiama l'attenzione sul diverso approccio al problema della creazione da parte dei teologi, «ai quali dovemo credere noi Cristiani», e dei filosofi naturali, in particolare dei «Peripatetici» ${ }^{19}$. Questa distinzione ritorna anche nella sopracitata lezione decima, ove Varchi approfondisce alcuni dei temi toccati nel commento letterale. A suo avviso, infatti, questo commento non è sufficiente: «fornita la sposizione», sarebbe stato opportuno «disputare alcune quistioni». Bersagliato dalle critiche di quanti sostenevano, in modo a suo giudizio infondato, che «nell'Accademia non si debbe favellare di cose filosofiche», Varchi decise però di rinunciare al metodo esegetico che avrebbe preferito: un metodo squisitamente scolastico, in cui all'expositio litterae doveva seguire la disputatio. Si risolse quindi ad adottare un procedimento diverso, presentato in questi termini, anch'essi di chiara ispirazione scolastica:

ho pensato $[\ldots]$ non di disputare, ma di recitare brevemente e dichiarare se non tutti quegli dubbi che per piena intelligenza di questo primo Capitolo si ricercavano, almeno quegli che mi sono paruti più necessari ${ }^{20}$.

Il primo di questi sette «dubbi» riguarda proprio la nozione di Dio come Primo Motore e pone l'interrogativo seguente: «perché volendo Dante significare Dio, dicesse: colui che tutto muove». Tale interrogativo è per Varchi

18. ENCD, vol. 28.4, p. 1564. Da Landino riprenderanno il parallelismo fra i due testi Trifon Gabriele e Bernardino Daniello. In proposito si veda Lombardo 2013, p. 500.

19. Varchi I, pp. 196-200; le citazioni sono dalle pp. 196, 199. Nel sottolineare che Dio dà essere e vita alle creature Varchi è in sintonia con quanto avevano sostenuto Francesco da Buti e Cristoforo Landino ad locum.

20. Varchi I, pp. 396-397. Già nelle lezioni sul venticinquesimo canto del Purgatorio Varchi si era mostrato consapevole dell'esigenza che l'insegnamento all'Accademia seguisse un metodo diverso da quello in uso nelle università: cfr. Varchi I, p. 98. 
ineludibile: considerato che Dio, di per sé ineffabile, è chiamato diversamente dai «poeti» e da «altri scrittori», ma anche dai «filosofi» e dai «teologi», non può stupire che «alcuni» si chiedano perché «Dante in questo luogo dove egli è teologo, volendo significare Dio, lo significò dal movimento e non da altra operazione e perfezione sua, come egli fece in molti altri luoghi, essendo il movimento appartenente non al metafisico, ma al filosofo na-

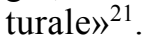

La risposta di Varchi ruota intorno al problema della dimostrabilità dell'esistenza delle sostanze separate e di Dio, e più precisamente intorno al problema di quale sia la scienza, aristotelicamente intesa, all'interno della quale tale dimostrazione è possibile:

devemo sapere, che egli fu ed è quistione grandissima non solamente tra' filosofi e teologi insieme, ma ancora tra i teologi medesimi ed i medesimi filosofi, a chi s'appartenesse di provare le sostanze separate, cioè che Dio e l'altre Intelligenze fussero, o al metafisico o al naturale ${ }^{22}$.

Elencati i commentatori greci, arabi e latini favorevoli alla prima ipotesi, Varchi sottolinea che «Averroè, quel grandissimo arabo, pruova il contrario, cioè che solo il filosofo naturale può dimostrare le sostanze astratte» ${ }^{23}$. Varchi coglie qui un'effettiva specificità di Averroè, che aveva preso le distanze da Avicenna secondo il quale la metafisica andava intesa come scienza dell'essere in quanto essere, come ontologia generale, e non come teologia, primariamente rivolta allo studio delle sostanze immateriali e di Dio. Per Avicenna infatti se la metafisica avesse Dio per suo soggetto proprio non potrebbe dimostrarne l'esistenza, dal momento che secondo Aristotele (An. Post., I, 10) nessuna scienza può dimostrare l'esistenza del proprio soggetto. Averroè aveva ribattuto che l'impossibilità di formulare prove dell'esistenza di Dio non rappresenta un limite della metafisica e non le impedisce di essere considerata come la scienza delle sostanze immateriali. Aristotele infatti aveva provato la loro esistenza all'interno di un'altra scienza, cioè nella física, e nel farlo, coerentemente con un'altra regola della sua epistemologia (An. Post., I, 9) secondo la quale una scienza non può dimostrare i suoi principi, aveva proposto argomenti di tipo induttivo: lungi dal procedere a una deduzione dell'esistenza di Dio, principio primo della natura, nella sua Fisica Aristotele era risalito dagli effetti alle cause, mostrando che l'esistenza di un primo moto nella natura, cioè l'eterna rotazione del cielo, comporta l'esistenza di un Primo Motore Immobile che, dotato di una forza infinita, non può che essere una forma immateriale ${ }^{24}$.

21. Varchi I, pp. 397-398.

22. Varchi I, p. 398.

23. Ibidem.

24. Per un'introduzione al problema si veda Di Giovanni 2017, pp. 207-210. Le critiche di Averroè alla concezione avicenniana della metafisica sono attentamente ricostruite da Bertolacci 2007. 
Varchi presenta ampiamente le «due ragioni potentissime» proposte da Averroè:

la prima delle qual è questa: Niuna scienza pruova mai che il subietto di lei sia, nè alcuna delle sue parti principali, anzi lo presuppongono tutte: il subbietto della Metafisica sono le sustanze astratte: dunque il metafisico non può provarle. La seconda è tale: Solo l'eternità del moto mostra che le sostanze siano, e l'eternità del moto appartiene al filosofo naturale: dunque solo il filosofo naturale dimostra le sostanze astratte; perciocchè se il moto è eterno, anche il mobile viene a essere eterno, dunque anche il motore sarà eterno altramente non potrebbe muovere in eterno $\mathrm{o}^{25}$.

Secondo Varchi la posizione di Averroè è corretta non solo dal punto di vista interpretativo, in quanto corrispondente all'autentico pensiero di Aristotele, ma anche dal punto di vista teoretico. Dante l'avrebbe condivisa e avrebbe aperto il Paradiso definendo Dio come «colui che tutto muove» proprio per sottolineare che la ragione umana può giungere a provare l'esistenza di Dio a partire da una riflessione sul movimento, quindi sul terreno della fisica e non della metafisica:

a noi bastarà sapere che, secondo Aristotile ed Averroè suo commentatore, solamente l'eternità del moto può provare il primo Motore essere astratto, e così non al filosofo divino, ma al naturale solo appartiene dimostrare le sostanze separate; e per questa cagione Dante, come grandissimo Peripatetico ed Averroista, disse:

La gloria di colui che tutto muover ${ }^{26}$.

Varchi chiarisce che il termine «tutto» non va frainteso, perché «il sentimento di Dante non è che tutte le cose si muovano, chè bene sapeva egli la divisione che fa Aristotile nell'ottavo della Fisica, cioè che delle cose alcune sempre si muovono, alcune sempre stanno ferme, alcune quando si muovono e quando stanno ferme»; quindi conclude che l'incipit del Paradiso vuole porre l'accento sul fatto «che tutte le cose che si muovono, si muovono dal primo Motore», il quale, come aveva ricordato poco prima, "è immobile e per se e per accidente $\rangle^{27}$.

Secondo Varchi si tratta della stessa tesi sostenuta, nei medesimi termini, da Severino Boezio. Abbiamo visto che già Pietro Alighieri aveva accostato il terzo verso dell'O qui perpetua del De consolatione philosophiae al primo verso del Paradiso, evitando però di segnalarne la chiara ispirazione aristotelica. Trifon Gabriele, Bernardino Daniello e Cristoforo Landino documentano quanto questa linea interpretativa fosse diffusa ancora nel Cinquecento, ma Varchi si spinge oltre, cogliendo la presenza di echi aristotelici non solo nell'incipit del Paradiso ma già nel carme boeziano. L'intuizione non è banale, considerato che questo carme è così ricco di spunti platonici che ancora

25. Varchi I, pp. 398-399.

26. Varchi I, pp. 399-400.

27. Varchi I, pp. 400-401. 
alcuni interpreti contemporanei lo leggono esclusivamente in questa chia$\mathrm{ve}^{28}$. Va detto però che Varchi si avvale dichiaratamente di una fonte che lo aiuta a interpretare la formula «stabilisque manens das cuncta moveri», cioè il commento al De consolatione philosophiae di «S. Tommaso» ${ }^{29}$. Oggi sappiamo che in realtà questo commento non è opera dell'Aquinate: secondo alcuni sarebbe attribuibile a William Wheatley - un maestro inglese formatosi a Parigi e attivo a Stamford nei primi decenni del XIV secolo - ma più probabilmente venne redatto nel XV secolo in area germanica ${ }^{30}$. Comunque sia, Brancato ha segnalato di recente che Varchi lo riprende alla lettera laddove si riferisce al Vangelo di Giovanni e alla sua interpretazione ${ }^{31}$. Si può aggiungere che, pur attingendo anche ad altre fonti, l'intera lettura del parallelismo Dante-Boezio proposta da Varchi dipende dallo Pseudo-Tommaso. Questi infatti scrive:

Notandum, quod Deus est stabilis, idest immutabilis, quia nulla specie motus movetur. Non generatione et corruptione, cum sit ingenerabilis et incorruptibilis. Nec augmentatione et diminutione, cum non sit quantus. Non alteratione nec loci mutatione, cum sit impartibilis. Et probat Aristoteles octavo physicorum, ex ordine moventium et mobilium necesse esse devenire ad aliquod primum omnino immobile et per se et per accidens.

[...].

Aliter exponitur: stabilisque manens das cuncta moveri: non quod omnia moveantur, sed quicquid movetur te dante movetur.

Et simile genus locutionis habetur joan. primo: "illuminat omnem hominem venientem in hunc mundum". Non quod omnes simul illuminentur: sed omnes qui illuminantur, a Deo illuminantur ${ }^{32}$.

28. Emblematico in questo senso Beierwaltes 1992. È curioso constatare come i commentatori medievali e rinascimentali che, da Pietro Alighieri in avanti, hanno accostato 1' $O$ qui perpetua all'incipit del Paradiso siano ignorati dai tanti dantisti contemporanei che insistono sulla sua ispirazione boeziana. È il caso fra gli altri di Ariani 2010, p. 186, che pure ritiene l'intera Cantica profondamente influenzata dal carme boeziano, che sarebbe «un vero e proprio palinsesto della macchina paradisiaca dantesca». Lombardo 2013 rappresenta una felice eccezione alla regola (a p. 656 il suo riferimento a «una consonanza basata sull'idea aristotelica del Primo mobile» è un lapsus per "Primo Motore").

29. Varchi I, p. 401.

30. La prima ipotesi, avanzata da tempo, è stata difesa di recente da Lucia 2012, che mostra interessanti paralleli fra il prologo del commento al De disciplina scholarium di William Wheatley e quello al De consolatione e ritiene che ciò rappresenti «una prova almeno favorevole all'attribuzione di entrambi i commenti al maestro inglese». Il commento sembra in realtà più tardo e proveniente dall'area germanica (cfr. Brancato 2018a, pp. 31-32), ma solo un'analisi approfondita della tradizione manoscritta potrà consentire di datarlo e contestalizzarlo meglio. Per una critica dell'interpretazone complessiva che Lucia offre del commento dello pseudo-Tommaso rimando a Bianchi 2016, pp. 242-245.

31. Cfr. Brancato 2018a, pp. 130-132.

32. Cito questo commento dall'edizione inclusa in $S$. Thomae Aquinatis Opera Omnia. Tomus XXIII.3, ed. Parma 1869, p. 81. 


\section{Afferma a sua volta Varchi:}

E se chi che sia dubitasse contra Dante, dicendo non esser vero che tutte le cose si muovano, come esempigrazia tutta la terra e il primo Motore stesso, il quale è immobile per se e per accidente, rispondiamo che il sentimento di Dante non è che tutte le cose si muovano, chè bene sapeva egli la divisione che fa Aristotile nell'ottavo della Fisica, cioè che delle cose alcune sempre si muovono, alcune sempre stanno ferme, alcune quando si muovono e quando stanno ferme; ma voleva dir Dante che tutte le cose che si muovono, si muovono dal primo Motore, nè poteva dinominarlo da operazione più manifesta che dal muovere, perché prima muove, poi mediante detto moto fa l'altre cose tutte quante. E questa medesima sentenza, con questo medesimo modo di favellare, disse il non men santo che dotto Boezio Severino in quel suo d'oro e veramente dottissimo e santissimo libro della Consolazione nella [sic] Filosofia, quando cantò:

O qui perpetua mundum ratione gubernas,

Terrarum coelique sator, qui tempus ab aevo

Ire jubes, stabilisque manens das cuncta moveri.

Il qual luogo interpretando S. Tommaso, allega quel passo del vangelo di S. Giovanni che dice: "qui illuminat omnem hominem venientem in hunc mundum", e lo espone, non che Dio illumini tutti gli uomini che vengono in questo mondo, ma che tutti quegli che sono illuminati, sono illuminati da lui; così il primo Motore non muove tutte le cose, ma muove tutte quelle che si muovono ${ }^{33}$.

In conclusione, come altri commentatori antichi Varchi enfatizza l'ispirazione boeziana del Paradiso. Tuttavia egli sostiene che, prima di essere "boeziano", Dante fu anzitutto un «grandissimo Peripatetico» e, più precisamente, un «Averroista». Se quattro secoli dopo Bruno Nardi avrebbe osservato che, ben presente in alcune opere filosofiche, l'averroismo nell'ultima Cantica aveva lasciato solo qualche "residuo", Varchi ritiene che esso condizioni la stessa immagine di Dio proposta sin dal primo verso: un Dio creatore che è anzitutto il Primo Motore Immobile, alla cui esistenza l'uomo, nella sua vita terrena, non giunge grazie alla speculazione metafisica ma tramite una più prosaica riflessione sulla mutabilità del mondo físico.

33. Varchi I, pp. 400-401. L'obiezione fondata sull'immobilità della Terra («se chi che sia dubitasse contra Dante, dicendo non esser vero che tutte le cose si muovano, come esempigrazia tutta la terra»), è abbastanza curiosa ma ha fondamento nella tradizione esegetica. Essa risale infatti al commento ad locum di Benvenuto da Imola (https://dante.dartmouth.edu/search_view.php?doc $=137553010010 \& \mathrm{cmd}=$ gotoresult\&arg $1=$ 8), «Et si obiicitur de terra quae est immobilis, dicendum est quod est verum quoad partem, non quoad totum»); ed era stata ripresa da Giovanni di Serravalle (https://dante.dartmouth.edu/search_view.php?doc $=141653010010 \& \mathrm{cmd}=$ gotoresult\&arg $1=$ 12): «posset tamen quis dicere: etiam terra non movetur, quia in medio omnium sita, in eternum stat. Respondetur, quod licet terra non moveatur quantum ad se totam, tamen eius partes moventur». 


\section{Benedetto Varchi, Averroè e le differenze intellettuali fra gli uomini}

Annalisa Andreoni ha pubblicato alcuni anni orsono un'eccellente monografia su Varchi che costituisce un punto di riferimento indispensabile per ogni ricerca sulla sua vita e le sue opere. Andreoni insiste a lungo, e giustamente, sul programma di volgarizzazione della filosofia che Varchi delineò e, in parte, realizzò nel corso del suo insegnamento a Padova e a Firenze. Nel farlo ella si spinge però a sostenere che ciò rivelerebbe - come anche altri studiosi hanno affermato - una «concezione della cultura orientata in senso fortemente "democratico" $\rangle^{34}$. Poco oltre Andreoni cita un passo della Lezione sopra le generazioni de' mostri, del 1548, nella quale Varchi asserisce che, una volta rimosse le barriere linguistiche, gli uomini «sono tutti da natura non pure desiderosi d'udire la verità delle cose, ma capevoli d'intenderla, solo che truovino chi possa loro, o voglia insegnarla ${ }^{35}$, quindi dichiara di non essere d'accordo con quanto da me sostenuto «sulla condivisione da parte di Varchi dell'idea di una distinzione antropologica di sostanza fra sapienti e indotti [...]. Tutta l'opera di Varchi dimostra il contrario» ${ }^{36}$.

Andreoni si richiama al mio saggio «Filosofi, uomini e bruti. Note per la storia di un'antropologia "averroista"», originariamente pubblicato nel 1992 su Rinascimento e nel 2003 incluso, in versione aggiornata, negli Studi sull'aristotelismo del Rinascimento ${ }^{37}$. È evidente che dedicando due paginette a Varchi ${ }^{38}$ non avevo la pretesa di offrire una ricostruzione esaustiva della sua antropologia ma volevo solo attirare l'attenzione su di un punto specifico. Un passo delle sue lezioni sul diciassettesimo canto del Purgatorio, risalenti al 1564, offre una testimonianza singolare dei possibili esiti del topos relativo alla "equivocità" del termine "uomo" predicato dei filosofi e dei non filosofi, che risale al gran commento al De anima e al cosiddetto "prologo all'ottavo libro" della Fisica di Averroè: un topos che, come il mio saggio cercava per la prima volta di documentare, venne variamente interpretato e utilizzato nel corso di tre secoli.

34. Andreoni 2012, p. 46. Di orientamento "democratico" e di "democratizzazione" della cultura in Varchi parlano anche Mikkeli 1999, p. 81, Sgarbi 2014, pp. 215-218, Dubard de Gaillarbois 2019, p. 54. Sgarbi 2017 insiste su questo tema, ma senza riferirsi esplicitamente a Varchi, di cui si limita a citare, a p. 74, il passo di cui qui sotto, n. 72. Perplessità in proposito esprime Giubilini 2015, p. 224, di cui condivido la tesi che «the intellectuals who participated in the activities of the earliest Academies not only belonged to the higher social ranks, who generally held the common people in contempt, but also included many university professors and students. The claims which are sometimes found in their translations and lectures should therefore be treated as rhetorical statements rather than declarations of intent».

35. Varchi III, p. 89. Cfr. Andreoni 2012, p. 50. Su questo passo si soffermano anche Siekiera 2007, p. 6, Sgarbi 2014, pp. 76-77 e Gilson 2018, p. 306, n. 118. Per la posizione di Varchi nel dibattito sulla generazione dei mostri rimando alla precisa analisi di Montemagno Ciseri 2007.

36. Andreoni 2012, p. 50, con la n. 27, corsivo dell'autrice.

37. Bianchi 2003, pp. 41-61. 
In effetti nel commentare i versi 91-105 («Né creator né creatura mai») del diciassettesimo canto del Purgatorio Varchi si sofferma sugli «animali razionali» - settimo dei dieci generi di sostanze di cui si compone l'intero universo - ed esalta, mescolando temi platonici, ermetici e aristotelici, il «miracolo» rappresentato dall'uomo, che «mediante gli abiti delle virtù e delle scienze, può copulare l'intelletto possibile coll'agente, cioè fare che siano un medesimo $»^{39}$. Egli afferma che «in questa copulazione consiste, secondo quel non mai bastevolmente lodato arabo Avenr, cioè figliuolo di, Rois, chiamato altramente Alulide Rosaceo, l'ultima perfezione, e per conseguente la suprema felicità e beatitudine umana $\rangle^{40}$. Slittando dal lessico averroista della copulatio e dell'ultima perfectio (che già Dante aveva ripreso ${ }^{41}$ ) a quello tipico della contemplazione religiosa, Varchi precisa che in questa condizione «si vede, s'intende e si fruisce l'ultimo bene intuitivamente e a faccia a faccia» (ove è facilmente percepibile l'eco di 1 Cor. 13,12$)^{42}$. L'uomo che grazie alle «virtù»e alle «scienze» raggiunge questa condizione, nuovamente descritta nei termini della noetica averroista della copulatio, realizza pienamente la sua condizione umana e al tempo stesso la supera, diventando «quasi Dio». Varchi ne trae la conseguenza - a mia conoscenza mai precedentemente formulata in termini così radicali - che

... per questo diceva quel medesimo Arabo, che uno uomo che sappia, e uno che non sappia sono equivoci, cioè si possono bene chiamare uomini, ma non sono: perchè hanno solamente il nome comune, ma non la sostanza ${ }^{43}$.

Che una simile affermazione evochi, letteralmente e senza alcuna presa di distanza, proprio l'idea di una «distinzione antropologica di sostanza fra sa-

38. Bianchi 2003, pp. 57-58.

39. Varchi I , pp. 146-147. Le due lezioni su questo canto risalgono al 27 agosto e 3 settembre 1564: cfr. Andreoni 2012, p. 318

40. Come segnala Nardi 1965, p. 327, n. 2 «Alulide Rosaceo» è «un curioso modo di umanisticizzare il nome arabo di Abu-l-Walīd Moḥammed ibn Aḥmed ibn Moḥammed ibn Rušhd».

41. Cfr. Bianchi 2018b.

42. L'intrecco fra il linguaggio averroista e quello paolino sulla visione "faccia a faccia" è presente anche nell'orazione funebre per Michelangelo Buonarroti, tenuta anch'essa nel 1564. Qui Varchi spiega che la «suprema felicità, e l'ultima beatitudine» dell'uomo consiste nell' 'intendere, amare, e fruire Dio perfettamente, e a faccia a faccia». In un simile contesto egli si allinea però all'ortodossia cristiana per sottolineare che questa condizione è raggiungibile solo nella vita ultraterrena e che quindi vi sono buoni argomenti filosofici e teologici per sostenere che, con la morte, Michelangelo ha conquistato «l'ultima perfezione, l'ultima felicità, e l'ultima beatitudine sua». Cfr. Varchi IV, p. 62. Su quest'orazione si veda Dubard de Gaillarbois 2019.

43. Varchi I, p. 148. L'autore di quella che, quando scrivevo, era la principale monografia sul Varchi aveva usato questo passo per esprimere un giudizio specularmente opposto a quello poi formulato da Andreoni e altri, cioè che «benché s'affaticasse a diffondere la cultura, non era sostanzialmente democratico»: si vedano Pirotti 1971, p. 89 e i miei rilievi critici in Bianchi 2003, p. 58, n. 38. 
pienti e indotti» mi pare semplicemente indiscutibile. Segnalo peraltro che non siamo in presenza di una tardiva sortita estemporanea. Dieci anni prima, nella lezione Della tragedia, Varchi aveva affermato:

... per questo diceva quel grande arabo secondo maggior segretario della Natura ${ }^{44}$, che un huomo Filosofo, e uno, il quale Filosofo non sia, sono equivoci, cioè non convengono tra loro in altro, che nel nome solo, come ancora un cavallo, benché sia o dipinto o morto, si chiama cavallo, e nondimeno altro di cavallo non ha, che la voce sola, cioè il nome di cavallo. E questo è quello che M. Giovanni Boccaccio non meno con dottrina, e giudizio, che con arguzia e leggiadria fece dire a M. Guido Cavalcanti singulare Filosofo, e poeta di quei tempi, nella nona novella della sesta giornata, quando egli sopraggiunto da messer Betto Brunelleschi, e altri di sua brigata tra quelle arche e sepolture di morti, che già in sulla piazza di santa Reparata presso la porta di San Giovanni si vedevano, e infestato sollazzevolmente da loro, che huomini idioti, e non letterati erano con importune dimande queste parole rispose: Signori voi potete dire a Casa vostra ciò che vi piace ${ }^{45}$.

Molto si potrebbe dire di questo passo, ove Varchi prima riprende da Averroè la tesi che il filosofo e il non filosofo sono uomini solo in senso equivoco, avendo in comune il «nome solo» ${ }^{46}$; poi chiarisce la nozione di equivocità a partire dalla distinzione - presente in Aristotele e sviluppata da Averroè - fra l'animale vivo e l'animale dipinto o morto ${ }^{47}$; infine collega genialmente questa

44. Questa definizione di Averroè si fonda ovviamente sull'implicita assunzione che il primo "segretario della natura" sia Aristotele: sulla genesi di quest'immagine dello Stagirita rimando a Monfasani 2016, XII, pp. 194-198.

45. Varchi III, p. 653. Servendosi di un lessico diffuso nella cultura medievale Varchi spesso contrappone gli uomini «dotti», «saggi» e «scienziati» agli «uomini idioti e volgari» (cfr. Varchi I, p. 11; Varchi II, p. 19), e in un caso distingue fra «dotti» e «laici» (cfr. Varchi II, p. 346). Varchi non attribuisce una valenza necessariamente dispregiativa agli aggettivi "idiota" e "volgare"; anzi, nel testo che si sta qui analizzando (Varchi III, p. 653), spiega poco oltre che non si deve presumere «che gli huomini idioti, cioè coloro che le lingue non sanno, non potessero essere ancora essi Filosofi, perché la Filosofia non nella cognizione delle lingue ma nella scienza delle cose consiste principalmente». L'ideale della "volgarizzazione" della filosofia - intesa come una forma di sapere che deve occuparsi delle cose e non delle parole - convive quindi con la tesi averroista che solo i filosofi realizzano pienamente la natura razionale propria dell'uomo.

46. Riferimenti ai limiti del definire qualcuno «equivocamente», «quanto al nome solo», ignorando differenze sostanziali si incontrano anche in altri passi di Varchi. Nell'orazione funebre per Michelangelo Buonarroti (Varchi IV, p. 41), Varchi critica quanti identificano il sapere filosofico e teologico con la formazione scolastica, «come se propriamente la Filosofia, e la sapienza non nella sostanza delle cose, ma ne' significati consistessero delle parole». Dopo aver distinto fra «dottori» e «dotti», egli sposta il discorso contrapponendo conoscenza teorica e concrete scelte di vita in questi termini: «Io non so se si chiamano, ma io so bene che non sono, e non doverrebbono essere chiamati Teologi (se non se equivocamente) ciò è quanto al nome solo, coloro, che hanno letto la scrittura divina, ma soli coloro, che quello osservano, e mettono ad essecuzione, che la scrittura divina ordina, e comanda».

47. Nel contesto toscano questa distinzione era stata applicata sin dal Quattrocento per presentare i dotti come soli "uomini veri”, come testimonia un passo delle Vite di Vespasia- 
distinzione alla celebre presentazione di Guido Cavalcanti nel Decameron (VI, 9): secondo Boccaccio, immerso nei suoi pensieri come si confà a un vero filosofo, Cavalcanti sarebbe stato importunato da una brigata di giovani spensierati e, con un'enigmatica ma folgorante battuta, avrebbe fatto prender loro coscienza che «a comparazione di lui e degli altri uomini scienziati» essi «e gli altri uomini idioti e non litterati» andavano considerati «peggio che uomini morti». Varchi dunque lascia intendere che Boccaccio avrebbe fatto esprimere a Cavalcanti, in una forma metaforica letterariamente efficace, proprio la tesi "averroista" secondo cui chi non esercita pienamente la propria razionalità non raggiunge la sua ultima perfectio, quindi conduce una vita che è tale solo all'apparenza. Per cogliere appieno la portata di quest'interpretazione, tanto audace quanto interessante, è indispensabile tenere in considerazione tre elementi. In primo luogo che, fosse o meno un "averroista", Cavalcanti aveva notizia, anche grazie ai suoi rapporti di amicizia con quel Giacomo da Pistoia che gli dedicò la sua Quaestio de felicitate, del dibattito in corso, a Parigi e in Italia, intorno all'idea che la realizzazione esistenziale e la felicità dell'uomo consistono nella contemplazione degli oggetti più nobili che si possano conoscere, cioè le sostanze separate e $\mathrm{Dio}^{48}$. In secondo luogo che per esaltare la filosofia come forma di vita più eccellente di ogni altra, l'unica davvero degna di essere vissuta, i magistri artium parigini del Duecento che la storiografia ha spesso etichettato come "averroisti" si erano effettivamente serviti di alcuni passi di Averroè, inclusi quelli nei quali si segnala che il predicato "uomo" si applica ai filosofi e ai non filosofi in modo equivoco. Infine che questi maestri, a iniziare da Alberico di Reims e Sigieri di Brabante, amavano però richiamarsi anche a una fonte diversissima, che è esattamente quella alla luce della quale, come ormai acquisito, il racconto di Boccaccio assume il suo pieno significato: la lettera di Seneca (Epist., 82, 3) nella quale la vita sine litteris è paragonata alla morte e alla vivi hominis sepultura ${ }^{49}$.

no da Bisticci (ed. A. Greco, vol. 2, p. 426), ove però manca significativamente ogni riferimento ad Averroè. Parlando di Benedetto Strozzi, Vespasiano sostiene infatti che il suo fu «un degno secolo di uomini docti, conversavano insieme et erano assai stimati da tutta la città per la loro virtù» e che «in quel tempo chi non sapeva lettere non era stimato fusse uomo, né nominato intra gli uomini degni, e che la differenzia che era tra uno che non sapessi lettere e uno le sapesse era quella è tra uno uomo dipinto e uno vero: quello è vero uomo che è letterato, quello non è vero uomo che è ignorante e sanza lettere. Sapesse la differenzia dall'uno all'altro lo iudicherebbe lui medesimo!».

48. Sull'averroismo di Cavalcanti ha richiamato l'attenzione Bruno Nardi, con un saggio che è stato poi ripreso e sviluppato da numerosi studiosi. Un ripensamento critico è stato avviato negli ultimi decenni: cfr. in particolare Falzone 2018 e Falzone 2019; ma si veda anche il contributo profondamente innovativo di Robert 2019. Sulla Quaestio de felicitate si veda in ultimo Zavattero 2005, che alle pp. 374-384 si sofferma sui rapporti fra Guido e Giacomo.

49. Cfr. Bianchi 1990, pp. 155-165 e 197, n. 98; Bianchi 2003, pp. 43-52, inclusa p. 46, n. 13. L'accostamento fra il passo in cui Seneca considera equivalente alla morte una vita sine litteris e la tesi risalente ad Averroè secondo cui gli ignoranti sono uomini solo in senso equivoco si ritrova già ben prima di Varchi - e prima di Boccaccio - nel prologo delle Questiones super librum Meteororum di Rodolfo il Bretone: «Sed propria operatio hominis 
Ritornando a Varchi è dunque un fatto che, due volte e a distanza di parecchi anni, egli riprende in termini abbastanza drastici il topos averroista della "equivocità" fra coloro che attualizzano e coloro che non attualizzano appieno le loro potenzialità razionali. Ciò ovviamente non significa che la sua concezione dell'uomo si riduca a questo. Al contrario, avendone lette innumerevoli varianti in molti pensatori operanti fra XIII e XVI secolo mi è ben chiaro che affermazioni di questo tenore non vanno prese troppo sul serio: osservo però che lo stesso può dirsi di tante rimasticature del tema della naturalità e universalità della sete di conoscenza risalente all'incipit della Metafisica («omnes homines naturaliter scire desiderant», secondo la vulgata dei florilegi aristotelici latini) che, in Varchi come in molti altri autori, devono essere valutate con prudenza. A mio modesto avviso «tutta l'opera di Varchi» dimostra che egli era fermamente convinto che le teorie filosofiche possono essere esaminate e discusse anche da chi non conosce le lingue nelle quali esse sono state originariamente formulate; che la mancata conoscenza del greco, del latino o dell'arabo non dovrebbe perciò impedire l'accesso al sapere, che è indispensabile strumento di progresso intellettuale e civile degli uomini; che è doveroso insegnare la filosofia, le arti e le scienze a un pubblico privo di formazione universitaria, diversamente da quanto fanno alcuni docenti che «insegnano prezzolati $\rangle^{50}$; che in determinate materie persone non istruite ma dotate di solida esperienza hanno idee più attendibili di tanti filosofi boriosi. Si tratta di convinzioni molto qualificanti, che evidenziano come Varchi, influenzato da figure come Dante, Pomponazzi, Achillini e Boccadiferro, fosse lontanissimo da quelle concezioni iniziatiche ed esoteriche del sapere che circolavano nella cultura del suo tempo ${ }^{51}$ e, in linea con tendenze radicate nella tradizione aristotelica latina sin da Alberto Magno, era pronto a privilegiare l'esperienza rispetto alle conoscenze puramente libresche dei filosofi. Così, per limitarsi a un

per quam distinguitur ab omnibus aliis animalibus est intelligere et scire sive operatio intellectus, quia in ista operatione intellectus hominis consistit eius felicitas, ut dicitur X Ethicorum, et per istam oprationem homo distinguitur a quolibet alio animali. Ergo, quando homo est perfectus in scientiis et maxime in scientiis speculativis, debet dici homo; cum autem non est perfectus in scientiis, sed est ignorans, non debet dici homo nisi equivoce. Et hoc dicit Seneca, in quadam epistula ad Lucilium: otium sine litteris mors est et vivi hominis sepultura. Sic ergo apparet quod homo ignorans non est homo nisi equivoce». Il prologo è analizzato e edito in Rossi 2011, cito da p. 379.

50. L'espressione è tratta dal Delle vie delle dottrine, edito in Andreoni 2012, p. 349.

51. Fondamentali qui le osservazioni presenti nel Proemio al commento all'Etica Nicomachea, edito in Andreoni 2012, pp. 331-333, poi svilupate nelle pagine iniziali della Lezione sopra quei versi del Trionfo d'Amore del Petrarca: cfr. Varchi II, pp. 17-19 (su cui si veda però qui sotto, n. 72); per la datazione rimando a Andreoni 2012, pp. 310-313. L'enfasi sugli esiti negativi della scelta di alcuni pensatori antichi di occultare «i divini misteri della santissima filosofia sotto diversi velamenti et varie figure» assume un significato preciso nella Firenze del Cinquecento, ove forte era l'influenza di concezioni iniziatiche ed esoteriche del sapere risalenti alla tradizione platonica e magico-ermetica. Sulla larga presenza di tali concezioni nella cultura del Rinascimento (e la frattura segnata su questo punto dal pensiero moderno) ha richiamato l'attenzione Paolo Rossi in un fondamentale saggio del 1977, ora riprodotto in Rossi 2006, pp. 227-267. Cfr. anche Perrone Compagni 2013. 
solo esempio, nello spiegare il venticinquesimo canto del Purgatorio Varchi riferisce che, secondo lo stesso Aristotele, sul problema della generazione e formazione del corpo umano è difficile raggiungere certezze, quindi continua:

anzi in questa, come in molte altre cose naturali, possono molte volte, e sogliono bene spesso intendere più, e giudicare meglio gli uomini idioti e volgari, che i dotti e scienziati. E però dovrebbero i filosofi in molte cose rapportarsi al giudizio di coloro i quali sono esercitati coll'opere tutto il tempo della vita loro in quello esercizio del quale essi scrivono a pena una volta colle parole; e io per me darei più fede in questo caso alle donne sperte, e anco a qualche uomo pratico, che a' filosofi, sì perchè la sperien$z a$ è in tutte le cose vera e certa maestra, e sì perchè questa materia, oltra l'essere incerta e dubbia da se, è stata trattata da tanti, in tante lingue e tanto diversamente che se io volessi arrecare insieme, non dico tutto quello che si potrebbe [...] ma tutto quello che n'è stato disputato, e pro e contra, da' più dotti e più approvati autori, sicuramente non basterebbero cento lezioni ${ }^{52}$.

Non credo però che tutto ciò basti a dare per acquisito l'orientamento "democratico" di Varchi e penso che per comprendere le sue convinzioni in materia di uguaglianza fra gli uomini si debba cercare di capire se l'ottimistica dichiarazione che essi «sono tutti da natura non pure desiderosi d'udire la verità delle cose, ma capevoli d'intenderla» ${ }^{53}$ sia confermata o meno da altri passi, precedenti e successivi. L'antropologia di Varchi è del resto assai complessa, anche perché risente di una pluralità di fonti letterarie, mediche e filosofiche. Risulta comunque chiaro che fra queste un ruolo di primo piano è svolto proprio dalle opere di un pensatore poco incline a sentimenti "egualitaristici" come Averroè. Sappiamo infatti che nell'intera produzione di Varchi, e in particolare nelle lezioni su Dante, i riferimenti ad Averroè abbondano ${ }^{54}$. Sappiamo inoltre che per il suo lavoro di filosofo, di commentatore di Aristotele (e, secondo un'opinione diffusa all'epoca, di traduttore) egli viene non solo ricoperto di sperticati elogi, ma è addirittura indicato come il protagonista decisivo nella translatio studii verso il mondo latino:

Alulido Rosaceo, chiamato comunemente Averrois, s'acquistò mediante la profondità della sua scienza e comento fatto da lui più che dottissimamente sopra tutte l'opere d'Aristotile, tanta autorità, che infino a pochi anni sono si leggeva ed allegava, per l'ignoranza delle buone lettere così greche come latine, non Aristotile, ma Averrois. E

52. Varchi I, p. 11.

53. Cfr. sopra, p. 635.

54. Nardi 1965, p. 323, ha individuato 67 citazioni di Averroè nelle opere di carattere filosofico di Varchi, 46 delle quali nelle sole lezioni su Dante. Elogi di Averroè si incontrano anche nella prima lezione sull'Etica Nicomachea e negli Scritti filosofici ora editi in Andreoni 2012, pp. 330, 340-341. Nardi 1965, pp. 381-382 e Garin 1989, pp. 142-148 hanno segnalato in Varchi la presenza della teoria dell'eterno ritorno, spesso associata all'insegnamento degli "averroisti”. Merita d'altra parte di essere rilevato che di recente Sgarbi 2015 ha mostrato che se si allarga la prospettiva di ricerca dalle edite lezioni su Dante ai manoscritti inediti di argomento psicologico si può cogliere che Varchi non accettò la tesi averroista dell'unità dell'intelletto potenziale. 
per vero dire se egli non avesse tradotto e comentato nella sua lingua arabica l'opere che si trovavano d'Aristotile, i Latini non avrebbono mai avuto Filosofia, perciocchè mediante quelle traduzioni che furono fatte poi d'arabo in latino, si cominciò a filosofare da dovero ${ }^{55}$.

Nell'ultima delle sue lezioni sul primo canto del Paradiso, prima di offrire l'originale interpretazione del primo verso sulla quale ci siamo soffermati sopra, Varchi si chiede perché fra tutte le creature solo gli uomini sembrino non desiderare o non poter conseguire «l'ultimo fine e beatitudine loro», come appare evidente considerato che «di tanto numero e quasi infinito pochissimi si dettero d'ogni tempo ed in ogni luogo o alle dottrine morali o alle discipline contemplative, e di quei pochissimi radi furono coloro che ne venissero a capo felicemente». Nel rispondere, Varchi ripropone, richiamandosi ancora una volta alle «verissime parole del grandissimo Averroè nel suo dottissimo proemio sopra il primo libro dell'Ascoltazione Naturale ${ }^{56}$, la teoria degli impedimenta con cui, dal XIII sino al XVI secolo, generazioni di aristotelici avevano cercato di coniugare la tesi che omnes homines naturaliter scire desiderant con la disincantata constatazione che la stragrande maggioranza di essi non ha la volontà né le capacità per appagare questo desiderio. Andreoni si sofferma su queste pagine ma cita integralmente solo il passo relativo al terzo di questi impedimenti, cioè l'ignoranza della logica:

La terza ed ultima ragione è il difetto ovvero mancamento della Loica, senza la quale, essendo ella lo strumento di tutte l'arti, come senza regolo o archipenzolo non può andarsi diritto se non a caso, non può sapersi cosa niuna certamente ${ }^{57}$.

Se questo è un impedimento superabile grazie a un'adeguata formazione, lo stesso non può dirsi dei primi due. Il secondo infatti consiste non solo (come ricorda Andreoni ${ }^{58}$ ) nei «mali costumi del suolo», ma anche nella «cattiva consuetudine», intesa in senso squisitamente averroista. Varchi infatti si richiama esplicitamente al «veramente divino proemio del terzo libro pure del-

55. Varchi I, p. 475. Poco oltre (p. 476) Varchi riconosce che negli scritti di Averroè, così com'erano a lui accessibili, si trovano «alcuni errori», che però attribuisce in parte ad errori di traduzione, sia dal greco all'arabo sia dall'arabo al latino; in parte al fatto che Averroè «fu uomo anch'egli e non Iddio». Si tratta ovviamente di un'applicazione al Commentatore del topos dell'umana fallibilità di Aristotele, diffusissimo fra medioevo e Rinascimento: cfr. Bianchi 2003, pp. 101-124 (con riferimento a questo passo di Varchi a p. 110, n. 20).

56. Varchi I, p. 393. Il rinvio al proemio della Fisica non pare particolarmente pertinente: gli argomenti presentati, come si vedrà più oltre, sono sviluppati maggiormente in altri luoghi dei commenti di Averroè.

57. Cfr. Varchi I, p. 395 e Andreoni 2012, p. 50. La convinzione che l'ignoranza della logica sia uno dei maggiori ostacoli all'acquisizione del sapere e della felicità è diffusissima nella cultura scolastica: per quanto riguarda Averroè si veda in particolare il gran commento alla Metafisica, II, 3, c. 15, ed. G. Darms, pp. 76, 78 (= ed. Giunta f. 35r B-C, F).

58. Andreoni 2012, p. 50. 
la Fisica» ${ }^{59}$ ove Averroè presenta la consuetudo come un ostacolo all'acquisizione della conoscenza scientifica, paragona la forza delle opinioni assorbite durante l'infanzia all'assuefazione ai veleni e attribuisce ai moderni loquentes, difensori dell'idea di creazione dal nulla, la tesi che lo studio della filosofia è incompatibile con quello delle "leggi", cioè delle religioni. Pur senza avventurarsi in interpretazioni troppo radicali di questo passo, spesso utilizzato per argomentare che chi ha avuto un'educazione religiosa non può più accedere alle verità filosofiche, Varchi insiste sul fatto che a lasciarsi condizionare da abiti mentali acquisiti, finendo per scambiare il vero col falso e viceversa, sono persino gli «uomini dotti e scienziati» e non solo, come prevedibile, la «gente volgare ed indotta»:

La seconda cagione è la cattiva consuetudine ed usanza, della quale favellò divinamente quel divino Arabo nel suo veramente divino proemio del terzo libro pure della Fisica, ed i mali costumi del suolo, nel quale nè la virtù nè le scienze nè si onorano nè si premiano... [...]. Nè si può agevolmente stimare quante siano e quanto grandi le forze della consuetudine, poiché l'uso non pure muta e converte la natura, come si dice ancora per proverbio, ma fa che le cose false sono credute ed amate come vere, e le vere rifiutate ed odiate come false, e questo non solamente dalla gente volgare ed indotta, il che sarebbe minor danno e meraviglia, ma bene spesso dagli uomini dotti e scienziati ${ }^{60}$.

Quanto al primo impedimento, esso consiste in una «indisposizione della natura», in una «complessione» che, secondo Varchi, non si limita a rendere temporaneamente inadatti alla "fatica del concetto" i «debili e infermi» (che Andreoni ricorda) ${ }^{61}$. In un passo molto significativo (che ella trascura), Varchi aggiunge che in seguito a come sono «composti e complessionati per la mala qualità e temperatura degli umori» certi uomini non sono capaci di comprendere i primi principi delle scienze, mentre altri non possono capirne le dimostrazioni e dubitano di cose evidenti alla ragione e ai sensi, oppure pretendono una certezza di tipo matematico anche in ambiti nei quali essa è impossibile ${ }^{62}$. Dopo aver concluso che «questi tali sono del tutto inetti e non idonei a filosofare», Varchi si appella a tre auctoritates - Galeno, che esalta sprecando superlativi, il De anima, citato implicitamente, e i Problemata pseudo-aristotelici - per sostenere che è indiscutibile che gli uomini si differenziano fra loro sulla base delle loro «complessioni». E benché a suo avviso ciò valga tanto sul

59. Per un'edizione di questo passo e un'analisi dei problemi filologici che esso ha posto si veda Schmieja 1986, pp. 175-184. Per un'analisi dell'uso che di esso si è fatto nel dibattito medievale e rinascimentale sul fondamento delle credenze religiose rimando a Bianchi 2018a.

60. Varchi I, pp. 394-395.

61. Andreoni 2012, p. 50.

62. Analoghe, ma assai più banali, sono le osservazioni sulle peculiari capacità di alcuni "tipi" di uomini: «come vedemo che ad alcuni uomini o dotti o ingegnosi basta loro solo accennare le cose, ad alcuni altri appena basta dichiararle loro». Cfr. Varchi I, pp. 501-502. 
piano intellettuale quanto su quello morale, una particolare enfasi è posta proprio sulle differenze di «ingegno»:

E non è dubbio nessuno, giudiziosissimi uditori, che tali sono gli uomini e quanto all'ingegno e quanto ai costumi, quali sono le complessioni loro, come dimostra dottissimamente il dottissimo Galeno in quel dottissimo libro dove egli pruova che i costumi dell'animo seguitano la temperatura del corpo $^{63}$; ed Aristotile medesimo non solamente dice che quegli che hanno la carne molle e delicata sono di buono ingegno ${ }^{64}$, ma afferma eziandio nei Problemi, che niuno fu mai in niuna o arte o scienza eccellentissimo, il quale non fusse malinconico di natura ${ }^{65}$, assomigliando i diversi e maravigliosissimi effetti della malinconia a' diversi e maravigliosissimi effetti del vino, come altra volta dichiararemo ${ }^{66}$.

Certo non siamo all'iperbole dell'inferiorità ontologica degli indotti, che secondo il sopracitato passo delle più tarde lezioni sul Purgatorio «si possono ben chiamare uomini» ma non lo sono «perché hanno solamente il nome comune, ma non la sostanza». Merita però di essere sottolineato che, come abbiamo visto, Varchi già un decennio prima aveva fatto ricorso ad Averroè per argomentare che filosofi e non filosofi «non convengono tra loro in altro, che nel nome solo»; e un ventennio prima, fornendo nell'ultima lezione sul primo canto del Paradiso la trattazione più approfondita del problema delle diverse attitudini intellettuali degli uomini, aveva insistito sull'esistenza di basi fisiopsicologiche che rendono alcuni per natura inadatti alla ricerca e alla contem-

63. Il riferimento è senza dubbio al trattato Quod animi mores corporis temperamenta sequantur, tradotto in latino da Niccolò da Reggio nel XIV secolo e poi ritradotto da svariati umanisti. Galeno è una delle auctoritates preferite da Varchi, che nelle lezioni sul venticinquesimo canto del Purgatorio (Varchi I, p. 101) non ha ritegno a presentarlo come un sostenitore della tesi, apertamente eterodossa, che l'anima è inseparabile dal corpo, è mortale ed è «qualità e temperatura ovvero complessione».

64. Cfr. De anima, II, 9, 421a25-26 (passo ripreso nelle Auctoritates Aristotelis, cfr. Hamesse 1974: «Molles carne mente aptos dicimus, duros vero ineptos»). Si tratta di un passo citatissimo, cui anche Tommaso d'Aquino si richiama di frequente per sostenere che alcuni uomini sono in grado di comprendere le stesse verità meglio di altri (si veda ad esempio la Summa Theologiae, I, q. 85, art. 7, resp.).

65. Cfr. Problemata, 30, 1, 953a10 (traduzione di Bartolomeo da Messina): «Propter quid omnes quicunque excellentiores fuerunt uiri. Aut secundum philosophiam, aut politicam, aut poesim aut artes uidentur melancolici esse: et hi quidem ita quod et occupantur egritudinibus que sunt a nigra colera». È notevole che questo passo sia citato nel commento al De consolatione philosophiae dello Pseudo-Tommaso che, come sappiamo, Varchi conosce e usa: «homines ergo melancolici maxime disponuntur ad magnas scientias acquirendas, et hoc propter multiplicationem plurium specierum: hinc que non absurde putandum est maiorem partem philosophorum melancholicos extitisse. Quod attendens philosophus libro problematum ait: quicumque excellentes fuerunt viri aut secundum philosophiam aut politicam aut poesim aut artes vocentur melancholici: etenim Hercules fuit hujus naturae, et occupantur ab aegritudinibus quae sunt a nigra cholera». Cito sempre dall'edizione inclusa in S. Thomae Aquinatis Opera Omnia. Tomus XXIII.3, ed. Parma 1869, p. 184.

66. Varchi I, pp. 393-394. Il tema del rapporto fra sapere e melanconia è ampiamente studiato e non è il caso di citare qui la ricchissima letteratura al riguardo. 
plazione della verità. Nel farlo, peraltro, egli molto significativamente si era richiamato sia al alcuni celebri passi del Commentatore, sia alla nozione di "complessione", che aveva fornito a generazioni di medici-filosofi del medioevo e del Rinascimento il fondamento teorico per argomentare due tesi. In primo luogo che gli uomini non partecipano allo stesso modo alla comune umanità, ma si differenziano sia a livello di popolazioni sia a livello individuale nella misura in cui una pluralità di fattori esterni (configurazioni astrali alla nascita, clima, alimentazione, età) plasmano e modificano gli equilibri umorali, quindi le attitudini comportamentali e intellettuali67. In secondo luogo che, di conseguenza, la tesi aristotelica della naturalità e universalità del desiderio di conoscere può e deve essere interpretata in senso fortemente elitario, riconoscendo che in realtà solo una piccola minoranza di uomini è dotata dei requisiti caratteriali e intellettuali indispensabili per soddisfarlo. Per limitarsi a un solo esempio fra i tanti possibili, il cosiddetto princeps Averroistarum Giovanni di Jandun - maestro operante a Parigi a inizio Trecento che Varchi conosce e che era una delle fonti del suo maestro Boccadiferro ${ }^{68}$ - aveva invocato proprio le differenze di complexio per spiegare perché, pur restando valido quanto affermato nell'incipit della Metafisica, molti uomini non possono in alcun modo raggiungere le verità metafisiche più profonde: essendo adatti solo «ad arare e alle scienze meccaniche», essi per Jandun non dovrebbero pretendere di «mangiare inutilmente il pane dei filosofi»» ${ }^{69}$.

Non c'è dubbio che Varchi avesse uno spirito meno esclusivo di Jandun: diversamente da quest'ultimo, e in sintonia con il suo amato Dante, riteneva auspicabile dare quel pane a un maggior numero di persone che sperava di raggiungere servendosi della lingua volgare ${ }^{70}$. Tuttavia, come lo stesso Dan$t^{71}$, Varchi ben sapeva che non erano solo le competenze linguistiche a ostacolare l'acquisizione del sapere, che poteva essere utilmente proposto ad alcuni, ma non a tutti. Non a caso, finita l'analisi dei tre motivi che rendono impossibile ai più un pieno appagamento del naturale desiderio di conoscere,

67. Fondamentale qui Chandelier-Robert 2013. È opportuno ricordare che Tommaso d'Aquino dovette dedicare un intero capitolo della Summa contra gentiles (II, c. 63) ad argomentare «quod anima non sit complexio, ut posuit Galenus».

68. Cfr. Varchi I, p. 115, ove «Giovanni Gandavense» è ricordato fra i «dottori latini».

69. Cfr. Bianchi 2013, pp. 350-354.

70. Ciò non significa che Varchi non trovasse sconveniente che questioni filosofiche complesse fossero affrontate da incompetenti. Significativa in proposito la sua irritazione di fronte al fatto che a Padova il problema del libero arbitrio fosse discusso non solo da filosofi e teologi, ma «da tutte le sorti dei letterati, anzi pure da qualunque idiota e vulgare uomo», compresi «i ciabattini e i fruttuaruoli, non che i sarti ed i calzolai»: cfr. Varchi I, pp. 385-386. Ma anche a proposito del rapporto anima-corpo Varchi precisa che esso può essere correttamente compreso dagli «essercitati, che gl'altri non possono intendere queste cose»: cfr. Varchi III, p. 501.

71. Mi riferisco a Convivio, I i 12, su cui Bianchi 2013, pp. 340-343, 351-352, ove si rinvia anche a Convivio, IV ii 7. Quest'ultimo testo dimostra che, come molti uomini del suo tempo, Dante credeva esistesse una precisa corrispondenza fra caratteristiche fisiche e capacità intellettuali: «La nostra mente ... è fondata sopra la complessione del corpo». 
Varchi afferma che in realtà molti uomini non vogliono o non possono apprendere, mentre quelli che "possono e vogliono» farlo sono pochissimi - e che quei pochissimi sono «sempre» stati i principali destinatari del suo insegnamento in qualità di console dell'Accademia Fiorentina:

Onde potemo brevemente conchiudere, mediante queste cagioni ed impedimenti, che degli uomini parte nè possono nè vogliono apparare, parte possono ma non vogliono, parte vogliono ma non possono, parte finalmente e possono e vogliono; ed a questi ultimi massimamente, radissimi senza alcun fallo, ma tanto più degni di qualunque più alta lode, ho io cercato sempre e con tutte le forze in questo mio consolato di soddisfare $^{72}$.

Una dichiarazione simile dovrebbe indurre a qualche pacata riflessione sugli obiettivi che Varchi si propose durante il suo magistero all'Accademia e sull'effettiva portata del suo programma di "divulgazione". Limitandosi al tema che stiamo qui esaminando, il minimo che si possa dire è che essa lascia ben poco spazio a letture "egualitaristiche" del modo in cui Varchi intende la società umana. Fedele a Aristotele, Varchi crede che gli uomini siano «da natura $[\ldots]$ desiderosi d'udire la verità delle cose» ma, anche se in una singola occasione si spinge ad affermare che tutti sarebbero anche «capevoli d'intenderla», nell'arco della sua intera carriera moltiplica asserzioni di segno opposto. Come si è appena rilevato, nel concludere solennemente la sua lettura del primo canto del Paradiso egli si sofferma a esaminare i motivi per cui la maggior parte degli uomini non ha accesso al sapere e constata - forse per ammonire, forse per lusingare il suo uditorio - che coloro che hanno la volontà e le

72. Varchi I, p. 395. La convinzione che la volontà e il talento indispensabile per conoscere non sono particolarmente diffusi è presente persino nelle pagine iniziali della lezione sul Petrarca, menzionate sopra alla n. 51, nelle quali Varchi prende le distanze dall'immagine esoterica del sapere. Di queste pagine talvolta si cita il passo (Varchi II, p. 19; cfr. Sgarbi 2017, p. 74) in cui si sottolinea il dovere morale di diffondere le conoscenze tramite un insegnamento chiaro, e in lingua volgare, capace di raggiungere un pubblico largo: «ciascuno prudente uomo e giudizioso debbe ingegnarsi con tutto il poter suo che gli idioti e volgari uomini siano meno che si possa, e ciò altramente non può farsi che con l'insegnare loro prima le virtù, poscia le scienze». Va però osservato che il passo continua così: «e se molti non vogliono o non possono, quando agevolissimamente s'insegnano, apprenderle, che dovemo credere che faranno, difficilmente insegnandosi?». La «forte tensione civile di queste parole» - per riprendere la felice espressione di Andreoni 2012, p. 315 - non esclude quindi il riconoscimento del fatto che, malgrado lo sforzo di chiarezza dei docenti, molti uomini non intendono né «possono» apprendere disposizioni morali e conoscenze che sarebbero loro indispensabili.

73. Diversa, ma degna di menzione la prospettiva delle lezioni sul secondo canto del Paradiso, del 1545 (per la datazione cfr. Andreoni 2012, p. 159). Commentando il v. 57 («la ragione ha corte l'ali»),Varchi insiste sui limiti della razionalità umana, fondata sulla conoscenza sensibile, e semplicemente registra il fatto che la ricerca della verità ha successo solo in pochissime occasioni: «Dovemo ancora sapere che la verità e la falsità delle cose non sono in esse cose nè ancora nei sensi propriamente ma nell'intelletto, il quale ha bene il vero per oggetto, ma non può sempre conseguirlo, anzi radissime volte, per queste ed altre ragioni dette altrove»: cfr. Varchi I, p. 461. 
capacità di imparare sono senza dubbio «radissimi» ${ }^{73}$. Ma prese di posizione analoghe si incontrano anche in due testi dedicati a temi diversissimi ma cronologicamente vicini. Nella questione Se $i$ calori come calori sono differenti fra sé, o pure sono tutti d'una medesima spezie spezialissima - dove l'auctoritas di Averroè è richiamata di frequente - Varchi attacca chi nega le evidenze sensibili, sostenendo che «chi nega il senso, non pur non è filosofo, ma né huomo»; evoca il principio aristotelico secondo cui non si può discutere «con chi non sa, o nega i principij, il che viene o dalla poca dottrina, e sperienza, o dalla molta perfidia»; quindi conclude:

E quanti sono coloro i quali non credono che cosa alcuna sia o vera o possibile, la quale essi o non sappiano o non habbiano veduta? E però non sono tutti gli huomini atti a filosofare, come havemo discorso altrove con più ragioni lungamente ${ }^{74}$.

Nell'ultimo capitolo dello scritto Di quei capi che $i$ greci chiamano prolegomeni o prototeorie ed $i$ Latini precognizioni ${ }^{75}$ Varchi addirittura liquida come una mera illusione l'ipotesi che «tutti gli uomini e tutte l'età siano atte ad imparare tutte le cose», indicando tre principali cause di questo fatto, registrato senza alcun imbarazzo: il «difetto di complessione», la mancanza di formazione logica, le cattive consuetudini acquisite. Anche in questo caso la pri$m a$ causa dell'impossibilità di accedere alle verità - e ai «piaceri diviniı ${ }^{76}$ della filosofia - viene individuata in una caratteristica puramente naturale, indipendente dalla volontà e dall'educazione dei singoli:

Non sia alcuno il quale si faccia a credere che tutti gli uomini e tutte l'età siano atte ad imparare tutte le cose: perciocchè alcuni per difetto di complessione, alcuni per lo non avere Loica, alcuni per essere male usati, ed alcuni altri per altre cagioni, non sono capevoli delle lettere, e massimamente della Filosofia ${ }^{77}$.

74. Varchi III, p. 258. Andreoni 2012, pp. 127-129 (ma cfr. anche p. 49) contesta con buoni argomenti che il testo derivi da una lezione accademica e lo mette in relazione alla Quistione sull'alchimia, mantenendo comunque una datazione al dicembre 1544, in linea con quanto risulta dalla epistola dedicatoria. Viene però da chiedersi a quale testo Varchi possa riferirsi quando rinvia a una sua precedente, approfondita analisi delle ragioni che spiegano perché non tutti gli uomini siano «atti a filosofare»: a mia conoscenza la più completa è quella della sopracitata lezione sul Paradiso, che è però della metà del 1545 .

75. Dario Brancato, che ringrazio per le comunicazioni personali sulla questione, ritiene che il trattatello sia databile fra 1545 e 1551 . Su di esso, e più in generale sugli scritti di logica e metodologia di Varchi, rimando a Papuli 1970, pp. 530-552; Sikiera 2013, pp. 145150 .

76. L'espressione si trova nel proemio alla lezione sul canto XXII del Paradiso, che sviluppa senza originalità i motivi classici della "felicità mentale" risalenti all'Etica Nicomachea: cfr. Varchi I, pp. 507-508.

77. Varchi II, p. 328. Varchi prosegue spostando l'attenzione sul rapporto fra conoscenza ed età, a partire dalle osservazioni di Aristotele che «diceva che un giovane non era uditore idoneo delle Morali, perché si lascia vincere dalle passioni, ed è troppo signoreggiato dagli affetti». Il riferimento è all'Etica Nicomachea, I, 3 . 
Non si può non apprezzare lo sforzo compiuto da Varchi per superare le barriere linguistiche che ostacolavano l'accesso alla cultura filosofica e il suo impegno per diffonderla, in aperto dissenso con quanti, ancora nell'Italia del Cinquecento, ritenevano che tramite «velami et copriture» si dovesse renderla inaccessibile agli «huomini idioti et volgari» ${ }^{78}$. Bisogna però prendere atto che, ispirandosi a una molteplicità di fonti mediche e filosofiche fra le quali spicca Averroè, Varchi ha, al contempo, sistematicamente difeso la tesi che fra gli uomini esistono differenze di «ingegno» costitutive e insormontabili. In più opere composte nell'arco di un ventennio egli ha ripetutamente affermato che all'interno della specie umana ci sono individui che hanno in comune con i sapienti solo «il nome» e che molti, a causa dell'ignoranza della logica, dei pregiudizi acquisiti ma anche e soprattutto della loro «complessione», sono «del tutto inetti e non idonei a filosofare», «nè possono nè vogliono apparare», non sono «atti a filosofare», non sono «capevoli [...] della Filosofia». Vi è una distanza abissale fra una simile concezione e quella che, all'inizio del secolo XVII, avrebbero assunto Francis Bacon, René Descartes, Thomas Hobbes e gli altri teorici della "eguaglianza delle intelligenze", concordi nel ritenere che a chiunque è dato acquisire il sapere purché segua un metodo adeguato. Nel 1634 Mersenne, dopo aver rilevato che i contadini e i nativi del Canada con le loro «gentilles inventions» dimostrano che la filosofia può essere appresa da soli, senza l'ausilio dei libri e dell'insegnamento dei «sçavans», affermerà che «un homme ne peut rien faire que l'autre ne le puisse semblablement. Et puis chacun contient en soy tout ce qui est necessaire pour philosopher, et pour raisonner de toutes les autres choses $\gg{ }^{79}$. La lunga stagione dell'antropologia "averroista" era ormai finita.

\section{Riferimenti bibliografici}

- Andreoni 2012: Annalisa Andreoni, La via della dottrina. Le lezioni accademiche di Benedetto Varchi, ETS, Milano 2012.

- Andreoni 2014: Id., Luoghi aristotelici nelle lezioni accademiche di Benedetto Varchi, in David A. Lines, Eugenio Refini (a cura di), Aristotele fatto volgare”. Tradizione aristotelica e cultura volgare nel Rinascimento, ETS, Pisa 2014, pp. 61-76.

- Ariani 2010: Marco Ariani, "Lux inaccessibilis". Metafore e teologia della luce nel Paradiso di Dante, Aracne, Roma 2010.

- Azzetta 2016: Epistola XIII, a cura di Luca Azzetta, in Dante Alighieri, Epistole. Egloge. Questio de aqua et terra, a cura di Marco Baglio, Luca Azzetta, Marco Petoletti, Michele Rinaldi, Salerno Editrice, Roma 2016, pp. 271-487.

- Azzolino 1839: Pompeo Azzolino, Introduzione alla storia della filosofia italiana ai tempi di Dante, s.e., Bastia 1839.

78. Queste formule sono tratte dal già citato proemio al commento all'Etica Nicomachea, edito in Andreoni 2012, p. 332. 254.

79. Mersenne 1634, question XXX, pp. 135-136: il passo è segnalato da Rossi 2006, p. 
- Barbi 1890: Michele Barbi, Dante nel Cinquecento, «Annali della Regia Scuola Normale Superiore di Pisa. Filosofia e Filologia», VII (1890), pp. 1-407.

- Beierwaltes 1992: Werner Beierwaltes, Consolazione nel concetto, in Id., Pensare l’Uno, Vita e Pensiero, Milano 1992, pp. 276-288.

- Bertolacci 2007: Amos Bertolacci, Avicenna and Averroes on the Proof of God's Existence and the Subject-Matter of Metaphysics, «Medioevo», XXXII (2007), pp. 61-97.

- Bianchi 1990: Luca Bianchi, Il vescovo e i filosofi. La condanna parigina del 1277 e l'evoluzione dell'aristotelismo scolastico, Lubrina, Bergamo 1990.

- Bianchi 2003: Id., Studi sull'aristotelismo del Rinascimento, Il Poligrafo, Padova 2003.

- Bianchi 2004: Id., Fra Ermolao Barbaro e Ludovico Boccadiferro: qualche considerazione sulle trasformazioni della "fisica medievale" nel Rinascimento italiano, «Medioevo», XXIX (2004), pp. 341-378.

- Bianchi 2011 : Id., Ludovico Boccadiferro, commentateur du De generatione, in Joëlle Ducos, Violaine Giacomotto-Charra (éd.), Lire Aristote au Moyen Âge et à la Renaissance. Réception du traité Sur la génération et la corruption, Honoré Champion, Paris 2011, pp. 249-258.

- Bianchi 2013: Id., "Noli comedere panem philosophorum inutiliter": Dante Alighieri and John of Jandun on Philosophical "Bread", «Tijdschrift voor filosofie», LXXV (2013), pp. 335-355.

- Bianchi 2015: Id., L'averroismo di Dante: qualche osservazione critica, «Le tre corone. Rivista internazionale di studi su Dante, Petrarca, Boccaccio», II (2015), pp. 71109.

- Bianchi 2016: Id., "Vae vobis homines": una massima di "Avenzoreth" e le sue metamorfosi fra XIII e XVI secolo", in Anna Rodolfi (a cura di), "Ratio practica" e "ratio civilis". Studi di etica e politica medievali per Giancarlo Garfagnini, ETS, Pisa 2016, pp. 225-248.

- Bianchi 2018a: Id., "Nulla lex est vera, licet possit esse utilis". Averroes" "Errors" and the Emergence in the Latin West of subversive Ideas about Religion, in Andreas Speer, Maxime Mauriège (hrsg.), Irrtum - Error - Erreur, De Gruyter, Berlin-Boston 2018, pp. 325-347.

- Bianchi 2018b: Id., "Ultima perfezione" e "ultima felicitade”. Ancora su Dante e l'averroismo, in Luca Bianchi, Onorato Grassi, Cecilia Panti (a cura di), Edizioni, traduzioni e tradizioni filosofiche (secoli XII-XVI). Studi per Pietro B. Rossi, Aracne, Canterano 2018, vol. 1, pp. 315-328.

- Bianchi 2019: Id., “Acte pur”: Dante, l'averrö̈sme et les substances séparées, in de Libera, Brenet, Rosier Catach 2019, pp. 307-330.

- Brancato 2018a: Dario Brancato, Il "Boezio" di Benedetto Varchi. Edizione critica del volgarizzamento della Consolatio philosophiae (1551), Olschki, Firenze 2018.

- Brancato 2018b: Id., Varchi e Aristotele. Nuovi materiali per il commento agli Analytica Priora, «Nuova Rivista di Letteratura Italiana», XXI (2018), pp. 99-155.

- Chandelier-Robert 2013: Joël Chandelier, Aurélien Robert, Nature humaine et complexion du corps chez les médecins italiens de la fin du Moyen Âge, «Revue de synthèse», CIIIIV (2013), pp. 473-510.

- Corti 1981: Maria Corti, Dante a un nuovo crocevia, Sansoni, Firenze 1981.

- Devlieger 2005: Lionel Devlieger, Benedetto Varchi on the Birth of Artefacts. Architecture, Alchemy and Power in Late-Renaissance Florence, tesi di Ph.D. presentata alla Universiteit Gent, Gent 2005.

- Di Giovanni 2017: Matteo Di Giovanni, Averroè, Carocci, Firenze 2017. 
- Dubard de Gaillarbois 2019: Frédérique Dubard de Gaillarbois, Michelangelo poeta e filosofo: un "invenzione" varchiana, in Selene Maria Vatteroni (hrsg.), La cultura poetica di Benedetto Varchi, Freie Universität Berlin 2019, DOI 10.17169/refubium 25666.

- ENCD: Edizione Nazionale dei Commenti Danteschi, Salerno Editrice, Roma 2001 ss. - Falzone 2013: Paolo Falzone, Bruno Nardi's Leuven Degree Dissertation (1911) and the Uneasy Character of Dante's Philosophy, «Tijdschrift voor filosofie», LXXV (2013), pp. 357-373.

- Falzone 2018: Id., Dante e l'averroismo di Cavalcanti: un nodo storiografico da ripensare? «Studi danteschi», LXXXIII (2018), pp. 267-306

Falzone 2019: Id., L'averroïsme du "premier ami” de Dante? Relecture critique d'une vulgate historiographique, in de Libera, Brenet, Rosier Catach 2019, pp. 235-268.

- Fioravanti 1966: Gianfranco Fioravanti, Boezio di Dacia e la storiografia sull'averroismo, «Studi medievali», VII (1966), pp. 283-322.

- Fioravanti 2019: Id., Dante et l'historiographie de l'averroïsme, in de Libera, Brenet, Rosier Catach 2019, pp. 403-417.

- Garin 1989: Eugenio Garin, Umanisti, artisti, scienziati. Studi sul Rinascimento italiano, Editori Riuniti, Roma 1989.

- Giglioni 2013: Guido Giglioni, Introduction, in Id. (ed.), Renaissance Averroism and its Aftermath: Arabic Philosophy in Early Modern Europe, Springer, Dordrecht 2013, pp. 1-34.

- Gilson 2012: Simon Gilson, "Aristotele fatto volgare" and Dante as "Peripatetico" in Sixteenth-Century Dante Commentary, "L'Alighieri. Rassegna dantesca», LIII (2012), pp. 31-63.

- Gilson 2016: Id., Vernacularizing Meteorology: Benedetto Varchi's Comento sopra il primo libro delle Meteore d'Aristotile, in Luca Bianchi, Simon Gilson, Jill Kraye (eds), Vernacular Aristotelianism in Italy from the Fourteenth to the Seventeenth Century, The Warburg Institute, London 2016, pp. 161-181.

- Gilson 2018: Id., Reading Dante in Renaissance Italy. Florence, Venice and the "Divine Poet", Cambridge University Press, Cambridge 2018.

- Giubilini 2015: Roberta Giubilini, Reassessing the Role of the Accademia degli Infiammati of Padua in the Evolution of Early Modern Logic, «Bruniana \& Campanelliana», XXI (2015), pp. 219-224.

- Gregory 1968: Tullio Gregory, Bruno Nardi, «Giornale critico della filosofia italiana», XVII (1968), pp. 469-501.

- Hamesse 1974: Jacqueline Hamesse, Les auctoritates Aristotelis. Un florilège médiéval. Étude historique et édition critique, Publications Universitaires de Louvain - Béatrice-Nauwelaerts, Louvain - Paris 1974.

- Hasse 2007: Dag N. Hasse, "Averroica secta”. Notes on the Formation of Averroist Movements in Fourteenth-Century Bologna and Renaissance Italy, in Jean-Baptiste Brenet (éd.), Averroès et les averroïsmes juif et latin, Turnhout, Brepols 2007, pp. 307-331.

- König-Pralong 2017: Catherine König-Pralong, La philosophie de Dante dans l'ombre de la Comédie: de Brucker à Witte, in Mario Longo, Giuseppe Micheli (a cura di), La filosofia e la sua storia. Studi in onore di Gregorio Piaia, Cluep, Padova 2017, vol. II, pp. 15-28.

- Imbach 1991: Ruedi Imbach, L'averroüsme latin du XIII siècle, in Ruedi Imbach, Alfonso Maierù (a cura di), Gli studi di filosofia medievale fra Otto e Novecento. Contributo a un bilancio storiografico, Edizioni di Storia e Letteratura, Roma 1991, pp. 191-208. 
- de Libera, Brenet, Rosier Catach 2019: Alain de Libera, Jean-Baptiste Brenet, Irène Rosier-Catach (éd.), Dante et l'averrö̈sme, Collège de France - Les Belles Lettres, Paris 2019.

- Lombardo 2013: Luca Lombardo, Boezio in Dante. La Consolatio philosophiae nello scrittorio del poeta, Edizioni Ca' Foscari, Venezia 2013.

- Lo Re 2008: Salvatore Lo Re, Politica e cultura nella Firenze cosimiana. Studi su Benedetto Varchi. Vecchiarelli, Roma 2008.

- Lucia 2012: Alessandro Lucia, "Unde Boetius in tractatu de summo bono dicit”. Il De summo bono di Boezio di Dacia nel commento di William Wheatley (XIV secolo) alla Consolatio Philosophiae di Boezio, «Studi medievali», LIII (2012), pp. 93-115.

- Marenbon 2013: John Marenbon, Ernest Renan and Averroism: The Story of a Misinterpretation, in Guido Giglioni (ed.), Renaissance Averroism and ist Aftermath: Arabic Philosophy in Early Modern Europe, Springer, Dordrecht 2013, pp. 273-283.

- Mersenne 1634: Marin Mersenne, Questions inouyes ou recreation des scavants, Villery, Paris 1634.

- Mikkeli 1999: Heiko Mikkeli, The Cultural Programmes of Alessandro Piccolomini and Sperone Speroni at the Paduan Accademia degli Infiammati in the 1540s, in Constance Blackwell, Sachicko Kusukawa (eds), Philosophy in the Sixteenth and Seventeenth Century: Conversations with Aristotle, Ashgate, Aldershot 1999, pp. 76-85.

- Monfasani 2016: John Monfasani, Greek Scholars between East and West in the Fifteenth Century, Ashgate, Farnham - Burlington 2016.

- Montemagno Ciseri 2007: Lorenzo Montemagno Ciseri, A lezione con i mostri. Benedetto Varchi e la Lezione sulla generazione dei mostri, «Rinascimento», s.s. XLVII (2007), pp. 301-345.

- Nardi 1965: Bruno Nardi, Studi su Pietro Pomponazzi, Le Monnier, Firenze 1965.

- Papuli 1970: Giovanni Papuli, B. Varchi: logica e poetica, in Studi in onore di Antonio Corsano, Lacaita, Manduria 1970, pp. 525-552.

- Perrone Compagni 2013: Vittoria Perrone Compagni, Parlare velato: trasmissione esoterica della verità nel Rinascimento, in Fabio Bazzani, Roberta Lanfredini, Sergio Vitale (a cura di), La verità in scrittura, Clinamen, Firenze 2013, pp. 73-86.

- Pirotti 1971: Ugo Pirotti, Benedetto Varchi e la cultura del suo tempo, Olschki, Firenze 1971.

- Renan 2002: Ernest Renan, Averroès et l'averrö̈sme, Maisonneuve et Larose, Paris 2002.

- Robert 2019: Aurélien Robert, Un averroïsme médical? Cavalcanti et Dante face à la passion amoureuse, in de Libera, Brenet, Rosier Catach 2019, pp. 269-305.

- Rossi 2006: Paolo Rossi, Il tempo dei maghi. Rinascimento e modernità, Cortina, Milano 2006.

- Rossi 2011: Pietro B. Rossi, Fra cielo e terra: Rodolfo il Bretone e la scienza meteorologica, in S. Perfetti (a cura di), Scientia, Fides, Theologia. Studi di filosofia medievale in onore di Gianfranco Fioravanti, ETS, Pisa 2011, pp. 357-386.

- Schmieja 1986: Horst Schmieja, Drei Prologe im grossen Physikkommentar des Averroes?, in Albert Zimmermann, Gudrun Vuillemin-Diem (hrsg.), Aristotelische Erbe im Arabisch-Lateinischen Mittelalter. Übersetzungen, Kommentare, Interpretationen, De Gruyter, Berlin - New York 1986, pp. 175-189.

- Sgarbi 2014: Marco Sgarbi, The Italian Mind. Vernacular Logic in Renaissance Italy (1540-1551) Brill, Leiden - Boston 2014.

- Sgarbi 2015: Id., Benedetto Varchi on the Soul: Vernacular Aristotelianism between Reason and Faith, «Journal of the History of Ideas», LXXVI (2015), pp. 1-23. 
- Sgarbi 2017: Id., Aristotle and the People. Vernacular Philosophy in Renaissance Italy, «Renaissance and Reformation», XXXIX (2017), pp. 59-109.

- Siekiera 2007: Anna Siekiera, Aspetti linguistici e stilistici della prosa scientifica di Benedetto Varchi, «Studi linguistici italiani», XXXIII (2007), pp. 3-50.

- Siekiera 2013: Id., L'eredità del Varchi, in Salvatore Lo Re, Franco Tomasi (a cura di), Varchi e altro Rinascimento. Studi offerti a Vanni Bramanti, Vecchiarelli, Manziana (Roma) 2013, pp. 145-171.

- Stabile 1991: Giorgio Stabile, Bruno Nardi storico della filosofia medievale, in Ruedi Imbach, Alfonso Maierù (a cura di), Gli studi di filosofia medievale fra Otto e Novecento. Contributo a un bilancio storiografico, Edizioni di Storia e Letteratura, Roma 1991, pp. 379-390.

- Varchi I: Lezioni sul Dante e prose varie di Bendetto Varchi la maggior parte inedite tratte ora in luce dagli originali della biblioteca Rinucciniana per cura e opera di Giuseppe Aiazzi e Lelio Arbib, Vol. I. Lezioni sul Dante, Società editrice delle storie del Nardi e del Varchi, Firenze 1841.

- Varchi II: Lezioni sul Dante e prose varie di Bendetto Varchi la maggior parte inedite tratte ora in luce dagli originali della biblioteca Rinucciniana per cura e opera di Giuseppe Aiazzi e Lelio Arbib, Vol. II. Prose varie, Società editrice delle storie del Nardi e del Varchi, Firenze 1841.

- Varchi III: Lezzioni di M. Benedetto Varchi Accademico Fiorentino [...] sopra diverse Materie Poetiche, e Filosofiche..., per Filippo Giunti, Firenze 1590.

- Varchi IV: Orazione Funerale di M. Benedetto Varchi fatta, e recitata da lui pubblicamente nell'essequie di Michelagnolo Buonarroti..., appresso i Giunti, Firenze 1564.

- Vasoli 2007: Cesare Vasoli, Benedetto Varchi e i filosofi, «Rivista di storia della filosofia», LXII (2007), pp. 1-25.

- Zavattero 2005: Irene Zavattero, La Quaestio de felicitate di Giacomo da Pistoia: un tentativo di interpretazione alla luce di una nuova edizione critica del testo, in Maria Bettetini, Francesco D. Paparella (a cura di), Le felicità nel Medioevo, Fédération Internationale des Instituts d'Études Médiévales, Louvain-la-Neuve 2005, pp. 355-409. 